\title{
The innovation strategy study of electrical engineering and automation specialty practice teaching
}

\author{
Limin Shao ${ }^{\text {a }}$, Meng Zhang ${ }^{\text {b }}$, Nan Wang ${ }^{c}$, Liai Gao ${ }^{d}$ and Jingya Li ${ }^{e, *}$ \\ College of Mechanical and Electrical Engineering, Agricultural University of Hebei, Baoding 071001, \\ China \\ ashaolm@126.com, b603691640@qq.com, ccmwn@163.com, dgaoliai111@126.com, \\ elijingya1965@126.com
}

\begin{abstract}
Practice teaching has an important position in engineering courses teaching. Cultivating innovative applied talents oriented market is the inevitable requirement of social development. As the popular specialty, electrical engineering and automation has higher quality requirement of the engineering practical ability, and employment rate is high. As a local university, connecting with the practical situation, Agricultural University of Hebei developed the “311” Talent-training Pattern, and constructed the professional practical ability training roadmaps. According to the roadmap requirement, it is imperative to reform the practice teaching of electrical engineering and automation. The reform objective is to cultivate innovative talents of "sound grounding, widening specialty, strong capability, emphasizing practice and high quality”. Relying on school laboratory, training center, and outside campus practical skills training base, it should student-centered and add comprehensive experimental contents. In addition, open laboratory, set up second class and participate in engineering practice actively. Through the cooperation with other companies, make the best use of resources. Pay attention to the combination of basic ability, specialty core competencies and expansional capacity, the practical ability and innovation consciousness of students has improved. Thus, it truly achieved the training objective of the compound talents.
\end{abstract}

Keywords: Electrical engineering and automation; practice teaching; "311" Talent-training pattern; roadmap; innovation consciousness.

\section{电气工程及其自动化专业实践教学创新策略研究}

\author{
邵利敏, 张梦, 王楠, 高立艾, 李静雅
}

河北农业大学机电工程学院, 保定 071001 中国

摘 要: 实践教学在工科专业教学中具有重要地位, 面向市场培养创新型应用人才是社会发展 的必然要求。作为热门专业, 电气工程及其自动化对工程实践能力要求较高, 就业形势也很 好。作为地方高校, 河北农业大学结合实际情况, 在 “311” 人才培养模式前提下, 绘制了本 科专业实践能力培养路线图。基于 “路线图” 的要求, 改革电气工程及其自动化专业实践教 学, 以培养 “厚基础、宽专业、强能力、重实践、高素质” 创新人才。以学生为主体, 以校 内外实验室、实训中心和实习基地为依托, 整合实验增加综合设计性实验, 开放实验室, 积 极参加工程科研实践, 以及通过校企合作等最大限度利用资源。注重基础能力、专业核心能 力和拓展能力相结合, 提高学生的实践动手能力, 强化培养学生的创新意识, 真正实现复合 型人才的培养目标。

关键词: 电气工程及其自动化; 实践教学; 人才培养模式; 路线图; 创新意识

\section{1. 前言}

高校是高素质人才培养基地。随着市场经济发展的日益完善, 高校的教育体制应与社会发展 和人才需求相适应。面向市场培养人才, 培养具有实践入门水平的工程师, 这是工科类本科 
教育的基本培养目标 ${ }^{[1]}$ 。提倡工程教育, 培养应用型人才, 提高工程师的职业素养和专业能 力, 必将对经济持续发展起到积极作用。作为工程教育的重要环节, 实践教学是提高人才培 养质量的重要途径, 实践是工程类专业的根本 ${ }^{[2]}$ 。这样以来, 改革实践教学是提高工程人才 培养水平的关键所在。实践教学环节能够促进知识结构、能力结构和科学思维产生交叉复合 作用, 在工程人才综合素质培养中占有极其重要的地位。加强实践能力, 能够有效的提高学 生分析问题、解决问题的能力, 对培养学生的创新意识至关重要。针对如何提升工科学生的 实践能力与创新意识, 培养应用型复合人才, 国内许多高校进行了大量的探索实践。河北农 业大学具有超百年的专业教学历史, “非试验不能探精微” 更是建校初期即提出的办学原则 [3]。由于学校历来重视实践教学, 注重学生创新能力的培养, 企事业用人单位对学校毕业生 满意度较高。在学校 “311” 人才培养模式指导下, 按照本科专业实践能力培养路线图, 探索 了电气工程及其自动化专业实践教学创新的有效策略。

\section{2. 实践教学改革的必要性}

河北农业大学于 2001 年开始招收电气工程及其自动化专业学生。秉承学校优良传统, 借鉴其 他院校在人才培养方面的成功经验, 以培养 “厚基础、宽专业、强能力、重实践、高素质” 创新人才为目标, 引进国内外先进教学方法和理念, 多次修订人才培养方案, 突出了实践教 学环节和创新环节的地位, 以提升学生的实践创新能力。河北农业大学电气工程及其自动化 专业旨在培养具有创新意识、综合素质高、适应能力强的具备地方电力系统及其自动化等有 关分析计算、工程设计、使用维护方面工作的高级应用型、复合型工程技术人才。

电气专业教学计划中具有相当多实践性较强的课程, 如电路、模拟电子技术基础、数字电子 技术基础、现代电气控制技术、电机与拖动、信号与系统、EDA 技术等，仅靠课堂理论讲授 远远不能满足要求, 必须加强实践教学环节提高实践创新能力。然而, 在实际教学过程中却 存在着许多不尽如人意的现象, 比如说, 有些学生调试电路时只要遇到问题, 不注意思考、 研究, 不是试图去排除故障, 而是 “积极” 向老师求援, 而老师不是有意识的去引导启发学 生, 告诫学生积极主动想办法解决, 而是亲自披挂上阵, 如此一来势必造成学生产生依赖性 [4]。另外, 学生操作顺序不规范, 无章可循, 不仅设备损坏多, 而且缺乏必要的安全意识, 这对将来工作安全生产是极其不利的。这些现象的存在, 老师和学生双方都应负有一定的责 任。必须大力提高学生实践创新能力, 毕竟企业单位不愿花费大量人力物力对应届毕业生进 行长期专门的岗位培训, 不仅增加用人成本, 也延缓学生实际就业时间, 甚至还有可能新人 跳槽为他人做嫁衣裳的危险。

\section{3. “311” 人才培养模式与实践能力培养路线图}

河北农业大学根据学校实际发展情况, 构建了 “ 311 ” 人才培养模式, 按照 “ $1+3+\mathrm{N}$ ” 模式构 建教学体系, 构建了 “一个中心、两个循环、三个层次” 的实践教学体系 ${ }^{[5-6]}$ 。从中很明显的 可以看到, 实践环节在河北农业大学的特殊地位。河北农业大学将科技管理领域的 “技术路 线图 (Technology Roadmap)” 理论引入到高等教育教学管理, 制定了本科专业实践能力培养 路线图 ${ }^{[7-9]}$ 。2013 年, “地方农业院校本科专业实践能力培养路线图的研究与实践”, 获得中 华人民共和国国家教学成果二等奖。电气工程及其自动专业如图 1 所示, 路线图的实施在一 定程度上可以解决工科学生实践动手能力差、创新能力不足等问题, 毕业生能够较好的满足 社会需求。

\section{4. 实践教学改革途径}

以校内外实验室、实训中心和实习基地为依托, 通过校企合作等最大限度利用资源, 提高学 生动手能力, 强化学生实践能力和创新意识培养。 


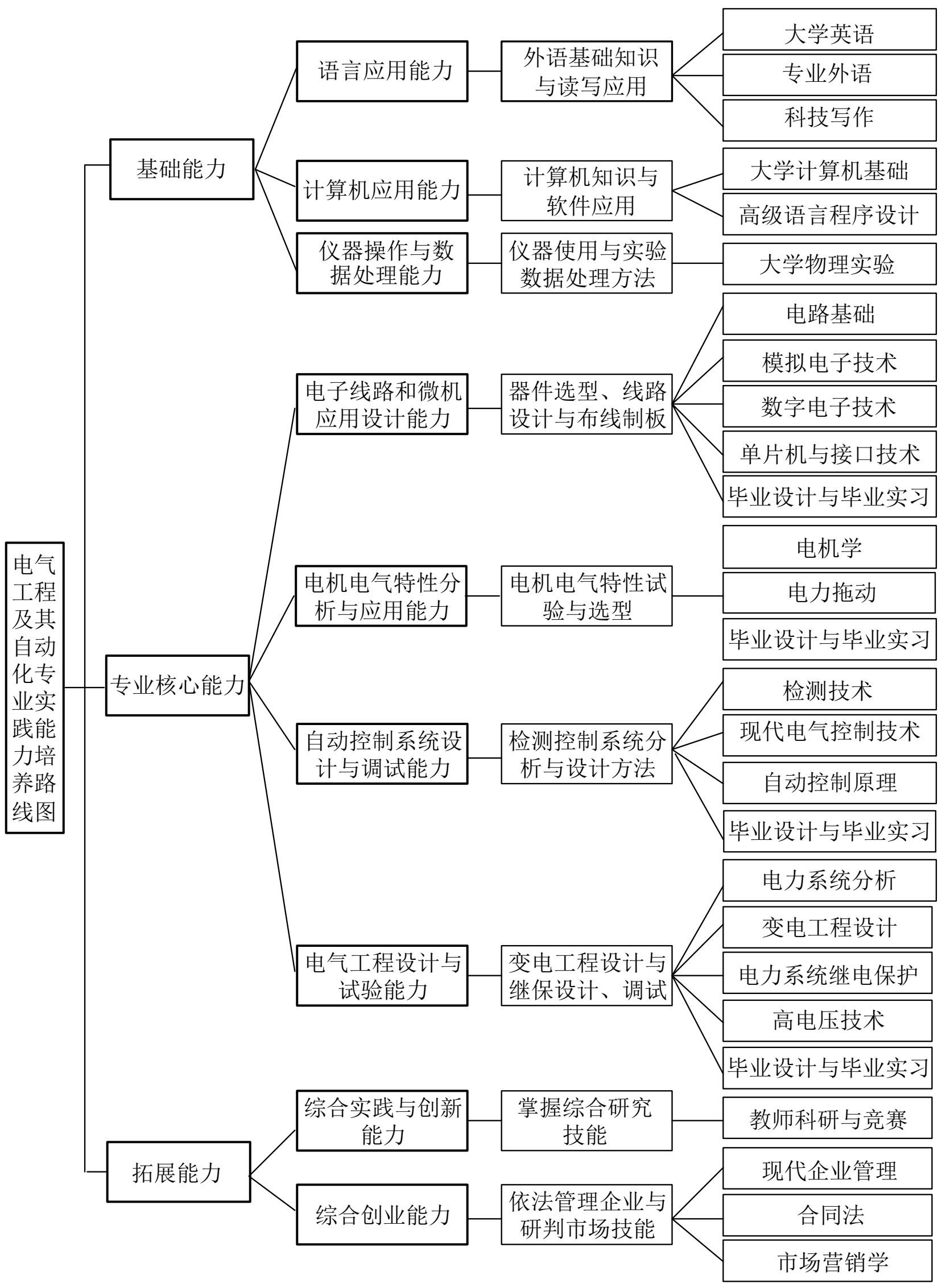

图 1 电气工程及其自动化专业实践能力培养路线图

4.1. 整合实验, 增加综合设计性实验

实验教学不只是对理论教学的单纯验证, 而应是教学环节的重要组成部分, 二者相互依存、 相互支撑。高等院校普遍重理论教学轻实验环节, 有限的实验学时只能用来安排验证性实验, 
学生缺乏实践训练机会, 一定程度上势必造成对学生实践能力培养的忽视。按照实践教学体 系要求, 整合实验, 减少验证性实验, 增加综合设计性实验, 条件成熟时实验单独设课。综 合设计性实验学时比例不少于总实验学时的 $80 \%$, 以切实可行性为原则, 可借助仿真软件, 指导教师发挥启发引导作用。通过综合设计性实验, 学生能够将所学理论知识和实验技能有 机结合, 用于解决较为复杂的实际问题, 培养独立自主解决问题的能力, 提高设计水平、动 手实践能力和创新意识, 综合素质得以提高。

4.2. 开放实验室

开放实验室, 将实验教学资源对学生开放。以学生为中心, 发挥学生的主体作用, 为其主动 获取知识、技能提供环境。开放实验室, 一方面能够充分发挥教学设备等资源的高效利用; 另一方面能够培养学生的自主学习能力, 提高其独立思考、分析问题和解决问题的能力。

4. 3. 积极参加工程科研实践

鼓励学生积极参加工程科研实践活动。所谓工程科研实践活动, 不仅是指教师的科研课题, 也包括课程设计和毕业设计等教学计划要求内容。应尽量取材于实际, 在保证先进性的同时 更要有实用性, 还得符合学校设备资源条件。只有这样, 才能较好的完成目的要求, 达到培 养综合设计能力的初衷, 能够使理论与实践有机结合。浓厚的科研学术氛围能够有效培养学 生的实践能力和创新精神。

4. 4. 积极参加竞赛项目

共青团中央、教育部和信息产业部等部门组织有一系列竞赛, 如全国大学生电子设计竞赛、

“挑战杯” 全国大学生课外学术科技作品竞赛等。可以说, 竞赛是实验教学环节的重要补充, 能够提高学生针对实际问题进行设计和制作的综合能力, 对提高学生的协作精神、实践动手 能力和创新能力都有很大的帮助。学校领导及教务处等相关部门高瞻远瞩, 充分认识到各种 竞赛项目对人才创新能力培养的重要作用, 已经连续多届资助电气专业学生参加电子设计竞 赛等各项赛事, 获奖多项, 数千学生从中受益。参赛学生的应变能力、创新能力以及协作精 神均得到了不同程度的提升, 增加了就业竞争力。

4. 5. 加强校内外实习基地建设

加强校内外实习基地建设, 学生通过参加实习基地的生产管理、经营管理和试验运行等方面 工作, 开阔了视野, 能够增强责任感 $[10]$ 。为此, 与河北农业大学后勤集团水电管理中心联系, 将学校西校区配电室、锅炉房等建设为电气工程及其自动化专业校内实习基地。除此之外, 还建设有保定热电厂、电力自动化研究所以及武强县供电公司等十多个国家电网分公司为校 外实习基地, 良好的校外实习基地能够很好的为学生实习提供服务。

\section{5. 教学评价}

现在, 高校许多学生普遍存在眼高手低问题, 不注意实践技能的培养, 实验纯粹是为应付了 事。很多同学在实验时只会 “照方抓药” , 几乎无法独立操作完成, 如果是当多人合作共同 完成的实验干脆一旁 “看热闹”。仅凭实验完成顺序与实验报告而给定实验成绩显然有失公 平, 很容易打消积极实验同学的积极性。因此, 必须制定合理的实验成绩评价体系。只有公 正客观的评价学生的实践能力, 同学们才能打心底里开始重视实验课, 进而有助于提高学生 参加实践环节的积极性。

\section{6. 结语}

作为地方院校, 电气工程及其自动化专业为河北农业大学工科热门专业。在学校 “311” 人才 培养模式指导下, 结合本科专业实践能力培养路线图, 研究构建了电气专业实践教学创新的 有效策略。充分尊重学生的主体地位, 通过增设综合设计性实验、积极参加工程科研实践和 竞赛等途径, 以就业为导向, 调动学生实践学习的积极性, 突出应用能力的培养, 提升学生 
的创新意识和创新能力。在今后的教学中还将继续探索改进, 努力培养符合专业培养目标和 社会需求的复合型应用人才。

\section{Acknowledgements}

This work is supported by the Agricultural University of Hebei the ninth batch of teaching research project (2015YB18).

\section{References}

[1]. Edward F. Crawley, Jianzhong Zha, Hohan Malmqvist, et al. On engineering education environment. (2008) No. 4, p. 13-21.

[2]. Shaoping Huang, Jing Li. Innovation practice teaching modes and training high quality engineering talents. Experimental technology and management. Vol. 29 (2012) No. 1, p. 18-20.

[3]. Zhigang Wang, Shuxing Shen, Hui Dang, et al. Practice and Thinking of “311” Talent-training Pattern. Higher Agricultural Education. (2005) No. 12, p. 3-5+28.

[4]. Jing Wang. Develop the comprehensive abilities of students through the experimental teaching of electrotechnics. China Modern Educational Equipment. (2007) No. 8, p. 72-73.

[5]. Zhigang Wang, Shuxing Shen, Yujian Zhai, et al. On the Practice of the Concept of "311" Talent-training Pattern. Journal of Agricultural University of Hebei (Agriculture \& Forestry Education). Vol. 10 (2008) No. 3, p. 245-247+251.

[6]. Limin Shao, Meng Zhang, Congcong Li, et al. Research of improving the electrical specialty student's experimental capability in high agricultural university. Journal of Chemical and Pharmaceutical Research. Vol. 6 (2014) No. 10, p. 743-746.

[7]. Li Zhang, Meng Zhang, Limin Shao, Dongming Li. Analysis of effective innovation strategies on electronic information engineering specialized practical teaching. Metallurgical and Mining Industry. Vol. 7 (2015) No. 6, p. 233-237.

[8]. Shuxing Shen, Yanyan Wu. Design and establishment for pratice teaching roadmap of agricultural university. Higher agricultural education. (2013) No. 7, p. 7-9.

[9]. Zhigang Wang. Resource intefration and construction of the specialty roadmaps of practical ability. China higher education. (2014) No. 5, p. 28-30.

[10]. Zhenhui Ren, Zhanlei Li, Yumei Sun, et al. Reseach on practice in teaching for electronic information specialty. Journal of Agricultural University of Hebei (Agriculture \& Forestry Education). Vol. 8 (2006) No. 4, p. 24-26. 